\title{
Protective Effects of Quercetin against Isoniazid and Rifampicin Induced Hepatotoxicity in Rats
}

\author{
Gulala Ibrahim Qader ${ }^{1}$, RoshnaShawkat Aziz ${ }^{1}$, ZheenAorahman Ahmed ${ }^{1}$, ZanaFaeq Abdullah ${ }^{2}$, Saad \\ Abdulrahman Hussain ${ }^{3, *}$ \\ ${ }^{1}$ Department of Pharmacology, School of Medicine, Faculty of medical Sciences, University of Sulaimani, Kurdistan, Iraq \\ ${ }^{2}$ School of Pharmacy, Faculty of Medical Sciences, University of Sulaimani, Kurdistan, Iraq \\ ${ }^{3}$ Department of Pharmacology and Toxicology, College of Pharmacy, University of Baghdad, Baghdad, Iraq \\ *Corresponding author: saad_alzaidi@yahoo.com
}

Received June 16, 2014; Revised July 03, 2014; Accepted July 08, 2014

\begin{abstract}
Drug-induced liver injury is a problem of increasing significance, but has been a long-standing concern in the treatment of tuberculosis (TB). The present study evaluates the possible hepatoprotective effects of Quercetin against the experimentally induced hepatotoxicity with isoniazid (INH) and rifampicin (RFP) in rats. Twenty-four rats wereallocated into 4 groups (6 rats in each group), and treated as follow: group I, received normal saline orally; group II, receivednormal saline + (INH 10mg/Kg/day and RFP $10 \mathrm{mg} / \mathrm{Kg} /$ day orally); group III, received Quercetin $(300 \mathrm{mg} / \mathrm{kg} / \mathrm{day})+(\mathrm{INH}$ and RFP as in group II);group IV, received N-acetyl cysteine (NAC) (50 mg/Kg/day) + (INH and RFP as in group II).After 42 days, the rats were scarified; blood samples obtained for evaluation of total antioxidant status (TAS), CRP, and the activities of ALT, AST, and ALP. Liver tissue sections were prepared for histopathologicalevaluation. The results clearly demonstrate that Quercetin provides significant protection against INH and RFP-induced toxicity in liver of rats, revealed as reduction in AST and ALT activities, increase in total antioxidant capacity, and improvements histopathologicalpicture of the liver. In conclusion, orally administered Quercetin protects the liver against INH and RFP-induced hepatotoxicity in rats.
\end{abstract}

Keywords: Quercetin, INH, rifampicin, hepatotoxicity, rats

Cite This Article: Gulala Ibrahim Qader, RoshnaShawkat Aziz, ZheenAorahman Ahmed, ZanaFaeq Abdullah, and Saad Abdulrahman Hussain, "Protective Effects of Quercetin against Isoniazid and Rifampicin Induced Hepatotoxicity in Rats.” American Journal of Pharmacological Sciences, vol. 2, no. 3 (2014): 56-60. doi: 10.12691/ajps-2-3-3.

\section{Introduction}

Toxic liver injury occurs more often than other organs, because all ingested substances that are absorbed are first presented to the liver and that the liver is responsible for the metabolism and elimination of many substances [1]. Many xenobiotics such as $\mathrm{CCl}_{4}$, isoniazid (INH) and Rifampicin (RFP) produce liver damage in a predictable and dose-dependent manner; INH and RFP are first-line drugs for treatment of tuberculosis (TB), and the hepatotoxicity associated with their long-term use remains a significant problem for their clinical use [2]. Peroxidation of endogenous lipids has been shownas a major mechanism involved in the hepatotoxic effects of INH and RFP [3]. This mechanism is generally attributed to the formation of highly reactive oxygen species (ROS), which act as initiators of lipid peroxidation and the consequent destruction and damage to the cell membrane [4]. Preventive care can significantly limit and reduce the progression of many types of liver diseases[5]. One of the drugs that used for this purpose is $\mathrm{N}$-Acetylcysteine (NAC), which is used clinically for the treatment of acute acetaminophen poisoning, manifested as progressive liver damage [6]. Oral supplementation with NAC provides an alternate means of boosting intracellular glutathione via elevated intracellular cysteine, and this can scavenge peroxynitrite and hydroxyl radicals as well as convert hydrogen peroxide to water [7]. Quercetin, the plantderived flavonoid, has a long history of traditional medicinal use in many countries. However, the flavonoids class themselves are not discovered until the 1930s. Currently, researchers focus on these naturally occurring substances and being a subject to many rigorous scientific and clinical studies. Quercetin in particular has shown great promise in a number of areas relevant to human health. Quercetin is a phenolic antioxidant with physicochemical properties that enable it to counteract the damaging effects of oxidation caused by ROS or other types of free radicals in many types of tissues and cells[8]. Oxidation reactions occur as part of the body's normal metabolic processes and also occur following exposure to environmental factors such as toxins in pollution, cigarette smoke, and fried foods. Quercetin's superior free radical scavenging capacity is believed to be one of the mechanisms by which it enhances endurance and performance, particularly insofar as research demonstrates that radicals and other ROS are an underlying aetiology in exercise-induced physiological disturbances $[9,10]$. The present study was designed to evaluate the possible 
protective effect of orally administered Quercetin against isoniazid and rifampin-induced hepatotoxicity in rats.

\section{Materials and Methods}

Twenty four 10 weeks old, male Sprague-Dawley rats weighing 150-200gm were housed in the animal house, School of Pharmacy, Faculty of Medical Sciences, University of Sulaimani in well ventilated plastic cages, at an ambient temperature $\left(25 \pm 2^{\circ} \mathrm{C}\right)$ and humidity of $55 \pm 5 \%$ under 12hr dark-light cycle, and allowed free access to water and food. Experimental protocols met the Guidelines for Animal Experimentation and approved by the Ethical Committee of the Faculty of Medical Sciences, University of Sulaimani. The animals were randomly allocated in to 4 groups (6 rats in each), and treated as follow: group I, the negative control, received $1 \mathrm{ml}$ of normal saline/day for 42 days; group II, the positive control, received $1 \mathrm{ml} /$ day saline by oral gavage tube for the first seven days; then, from the day 8 to day 42, the animals received oral daily doses of INH(SDI, Iraq) and RFP(SDI, Iraq) $(10 \mathrm{mg} / \mathrm{kg} /$ day for each one of them); group III, received oral doses of Quercetin (Xian Co, China) (75 mg/kg/day), with the daily doses of INH and RFP as in group II, starting from the first days and continued up to the day 42; group IV, the standard hepatoprotectant group, treated with a single daily dose of N-acetyl cysteine (Health Biotech Ltd., India) (NAC, $25 \mathrm{mg} / \mathrm{kg}$ ), administered orally by gavage tube for 42 days, with the daily doses of INH and RFP as in group II.On day 43, the animals were sacrificed andblood samples obtained by cardiac puncture in a plain tube; the samples were left to clot and centrifuged to obtain the serum, which is utilized for the estimation total antioxidant status (TAS), C-reactive protein (CRP), and the liver enzymes, AST, ALT, and alkaline phosphatase (ALP), utilizing ready-made kits (Randox, UK),according the specifications of manufacturer. Tissue samples of the liver were quickly existed and placed in formaldehyde, and processed later for histopathological evaluation [11].

\section{Statistical Analysis}

All the results were expressed as mean \pm SEM. Analyses were processed using Graph Pad Prism software for Windows (version 5.0, Graph Pad Software, Inc., San Diego, CA). The significance of difference among the studied groups was determined using one-way analysis of variance (ANOVA) followed by Bonferroni'spost hoc test. Values with $P<0.05$ were considered significant.

\section{Results}

Figure 1 clearly shows that the total antioxidant status (TAS) value was significantly decreased due to treatment with a combination of INH and RFP (group II) compared with untreated controls $(61.4 \%, P<0.05)$. Meanwhile, coadministration of either Quercetin or NAC with the antitubercular agents elevates TAS value in groups III and IV; the achieved values were comparable to that reported in control (group I). In Figure 2, treatment of rats with a combination of INH and RFP did not produce significant changes in serum levels of CRP $(P>0.05)$ compared with non-treated control animals. Moreover, co-administration of either Quercetin or NAC did not produce significant changes in serum CRP levels compared to both negative and positive controls, respectively $(P>0.05)$. Regarding the effects of long-term administration of anti-tubercular drugs on the serum levels of liver enzymes, Figure 3 demonstrates that treatment of rats (group II) with a combination of INH and RFP produces a highly significant increase in serum ALT activity $(167.4 \%, P<0.001)$ compared to untreated controls. Figure 3 also shows that co-administration of either Quercetin or NAC with the anti-tubercular drugs significantly attenuates the elevation of serum ALP activity levels, which appeared comparable to that reported in non-treated controls (group I). Similarly, Quercetin and NAC significantly attenuate the elevation in serum levels of AST activity that already elevated due to treatment with INH and RFP (180.4\%, $P<0.001$ ), and achieving full protection with AST values comparable to that reported in untreated controls (Figure 4). The results presented in Figure 5 clearly shows that longterm administration of INH and RFP produces slight elevation in serum ALP activity, which was not significantly different compared to untreated animals (9.5\%, $P>0.05)$. Meanwhile, co-administration of either Quercetin or NAC did not produce significant changes in serum ALP activity compared with either untreated or treated animals (groups I and II). In Figure 6, a section of liver tissue from rats treated with a combination of INH and RFP shows picture of liver toxic injury with mild degenerative changes in addition to focal lobular inflammation, eosinophils infiltration in the portal tracts and lobular parenchyma, with marked Kupffer cell hyperplasia and scattered apoptotic hepatocytes. In Figure 7, the section from liver of rats treated with Quercetin in addition to the anti-TB drugs for 42 days shows preserved liver lobular architecture with minimal Kupffer cell hyperplasia, with marked reduction in the number of eosinophils, in both portal tracts and lobular parenchyma; this was associated with notable absence of hepatocyte apoptosis. This indicates that Quercetin has notable hepatoprotective effect. Similarly, the section from liver of rat treated with NAC in addition to the anti-TB drugs for 42 days showspreserved liver lobular architecture comparable to that reported in animal group treated with Quercetin (group III) (Figure 8).

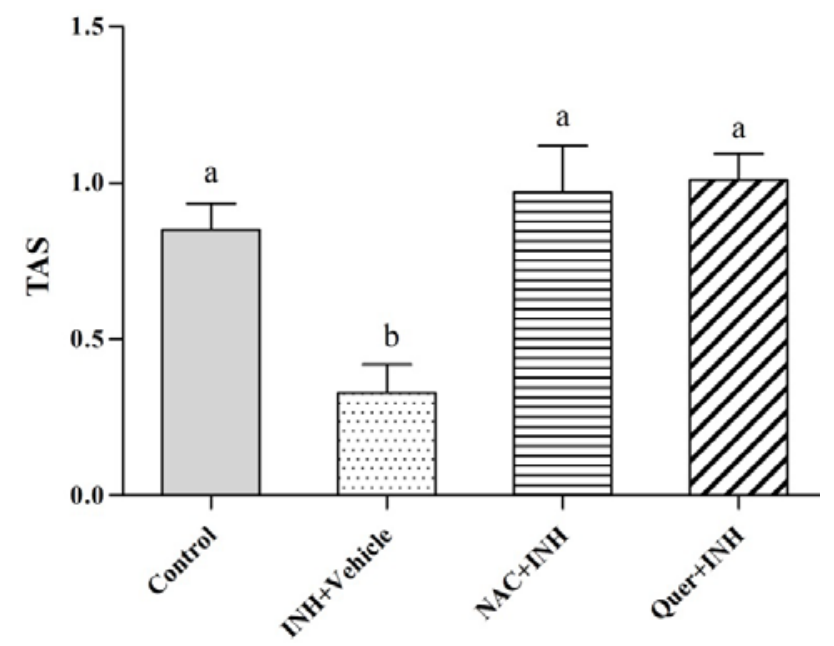

Figure 1. Effect of treatment with Quercetin or N-acetylcysteine on serum TAS level in rats treated with a combination of INH and RFP. Values are presented as mean \pm SEM; $n=6$ animals in each group; values with different letters $(\mathrm{a}, \mathrm{b})$ are significantly different $(P<0.05)$ using ANOVA and post hoc test 


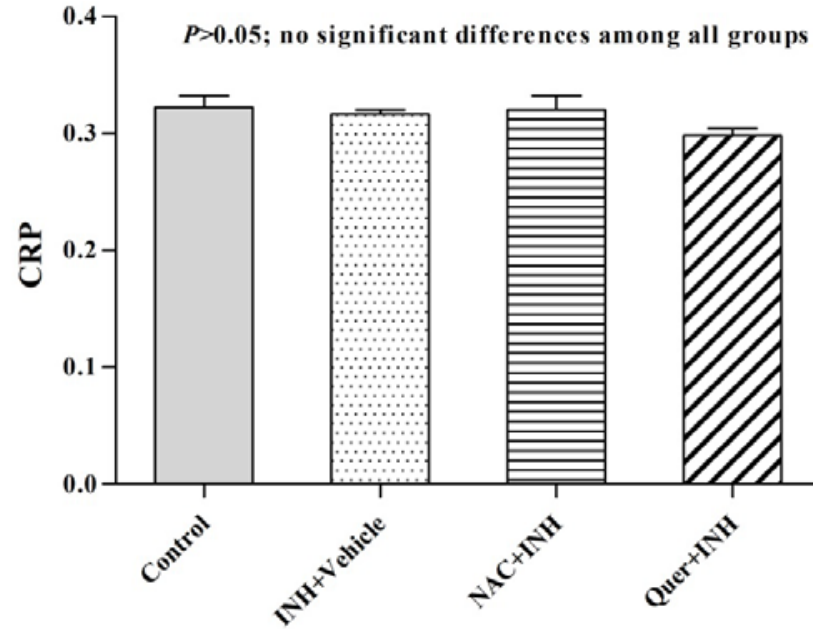

Figure 2. Effect of treatment with Quercetin or N-acetylcysteine on serum CRP level in rats treated with a combination of INH and RFP. Values are presented as mean \pm SEM; $n=6$ animals in each group; no significant differences $(P>0.05)$ reported among groups using ANOVA and post hoc test

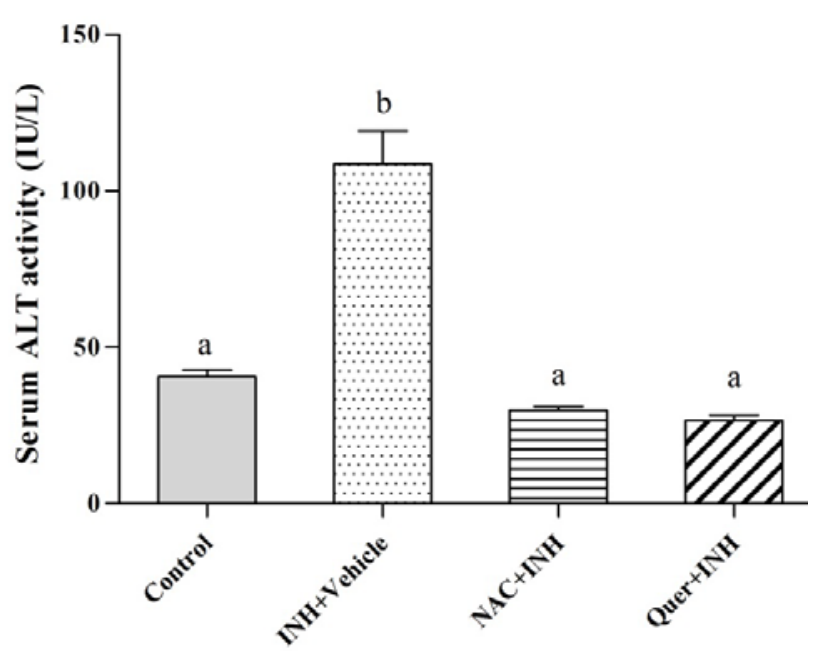

Figure 3. Effect of treatment with Quercetin or N-acetylcysteine on serum ALT level in rats treated with a combination of INH and RFP. Values are presented as mean \pm SEM; $n=6$ animals in each group; values with different letters $(\mathrm{a}, \mathrm{b})$ are significantly different $(P<0.05)$ using ANOVA and post hoc test

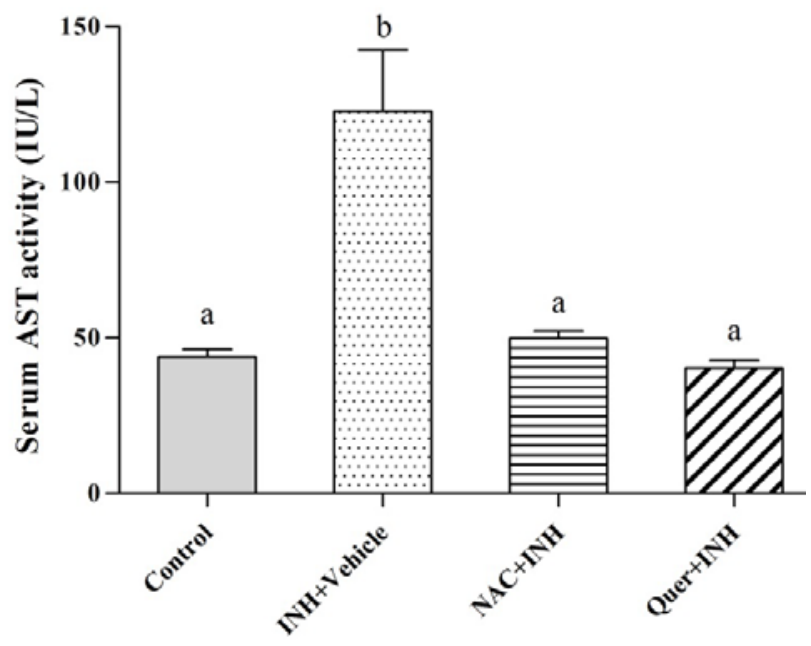

Figure 4. Effect of treatment with Quercetin or N-acetylcysteine on serum AST level in rats treated with a combination of INH and RFP. Values are presented as mean $\pm S E M$; $n=6$ animals in each group; values with different letters $(\mathrm{a}, \mathrm{b})$ are significantly different $(P<0.05)$ using ANOVA and post hoc test

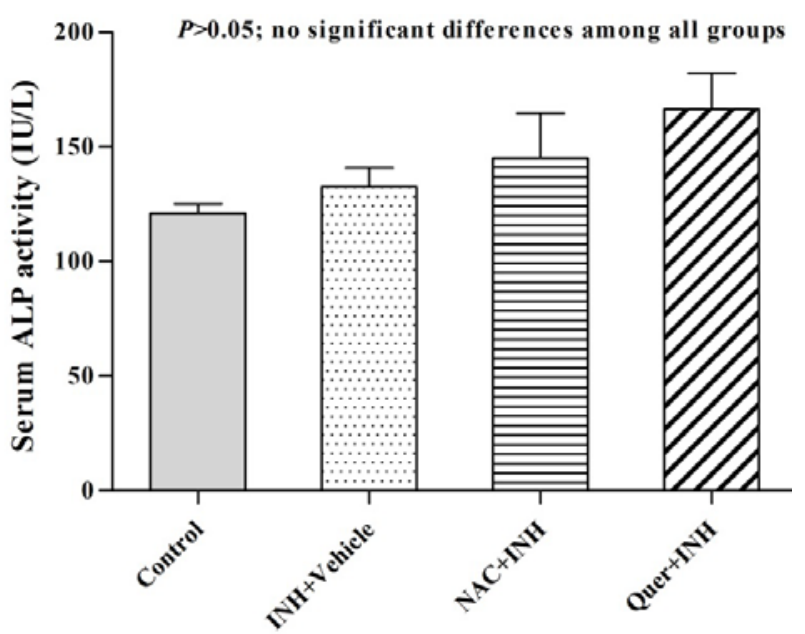

Figure 5. Effect of treatment with Quercetin or N-acetylcysteine on serum ALP level in rats treated with a combination of INH and RFP. Values are presented as mean $\pm S \mathrm{EM} ; \mathrm{n}=6$ animals in each group; no significant differences $(P>0.05)$ reported among groups using ANOVA and post hoc test

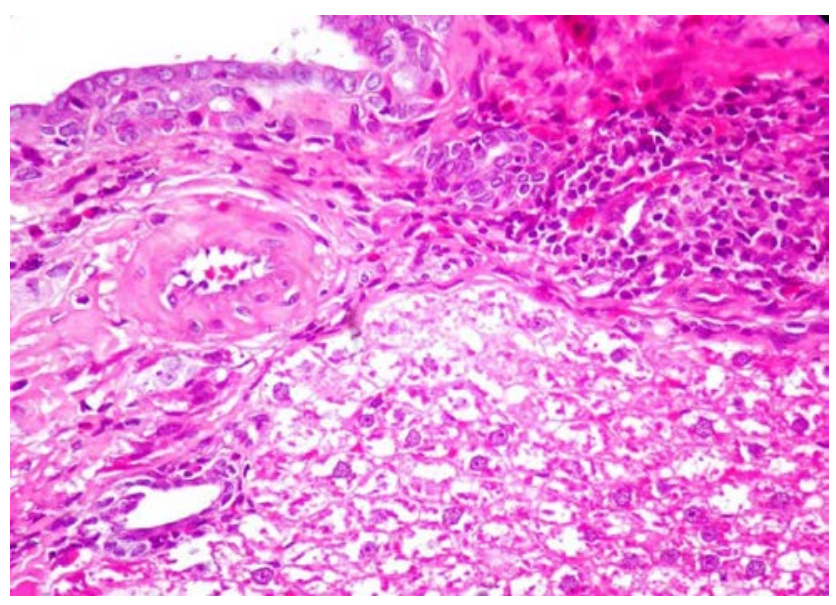

Figure 6. A portal tract in liver of a rat treated with INH and RFP for 42 days. A moderate mononulcear inflammatory cell infiltrate is noted in the portal tract with relative prominence of eosinophils (black arrows) (indicate drug-related hepatotoxicity). 400X magnification, H\&E stain

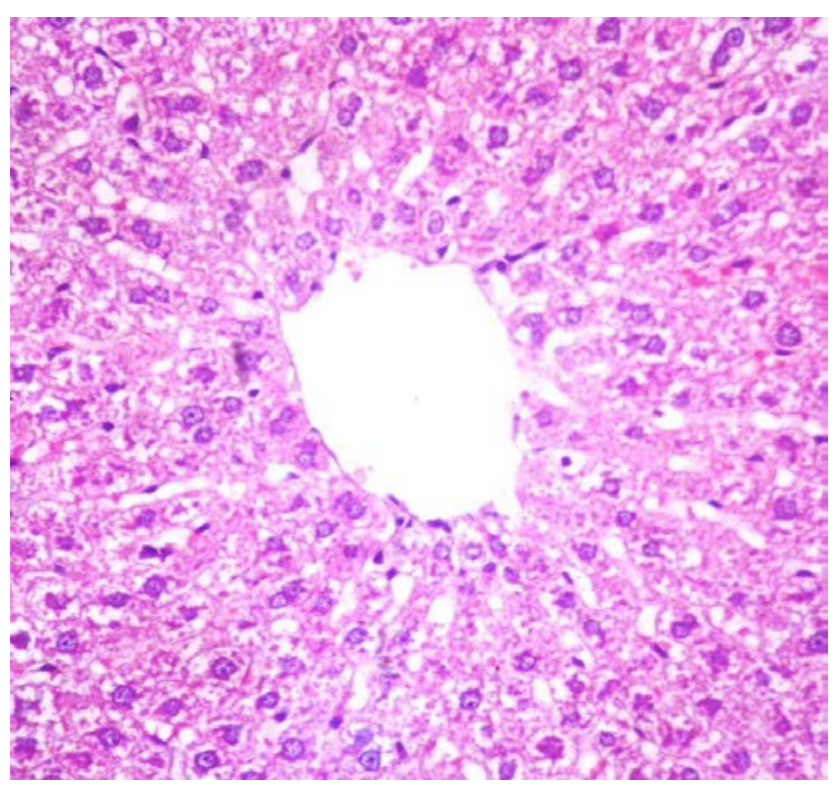

Figure 7. Sections of liver tissue from a rat treated Quercetin in addition to the anti-TB drugs (INH and RFP) for 42 days. The picture shows perivenular (zone-3) zone with minimal Kupffer cell hyperplasia (Black arrows). $100 \mathrm{X}$ magnification. H\&E stain 


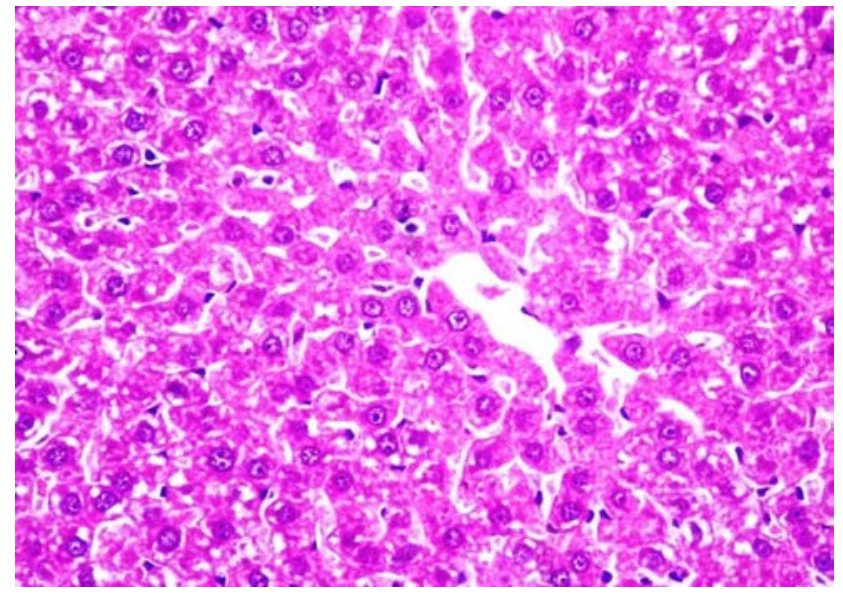

Figure 8. Sections of liver tissue from a rat treated NAC in addition to the anti-TB drugs (INH and RFP) for 42 days. There is a marked reduction of eosinophils in lobular parenchyma compared with liver from rats that have received anti-TB alone. However, there is still some degree of Kupffer cell hyperplasia(Black arrow). $100 \mathrm{X}$ magnification. H\&E stain

\section{Discussion}

Drug-related hepatotoxicity cannot be viewed as a single disease. Many different mechanisms lead to hepatotoxicity, including disruption of the cell membrane and cell death. This may result from covalent binding of the drug to cell proteins, which creates new adducts that serve as immune targets, thus inciting an immunologic reaction [12]. Other causes included inhibition of cellular pathways of drug metabolism [13], abnormal bile flow due to disruption of subcellular actin filaments and/or interruption of transport pumps, leading to cholestasis and jaundice with minimal cell injury [14], programmed cell death (apoptosis) through tumor necrosis factor and Fas pathways [15], and inhibition of mitochondrial function with accumulation of reactive oxygen species and lipid peroxidation, fat accumulation, and cell death [16]. Administration of INH and RFP produces different types of metabolic and morphologic aberrations in the liver because the liver is the main site for detoxifying these anti-tubercular drugs [3]. Increasing evidence suggests that these toxic metabolites can induce oxidative stress in the liver in rats $[17,18]$. Metushi et al. reported that INH itself could be oxidized to a reactive metabolite that covalently binds to hepatic proteins, and INH-induced liver injury is immune-mediated [19]. In the present study, we used an animal model to reveal the protective role of Quercetin against INH/RFP-induced hepatic toxicity. We found that INH and RFP treatment induced significant hepatic histopathological injuries, decreases TAS levels, increases serum CRP, AST and ALT levels, indicating the ability of such combination to induce hepatic oxidative stress. In the biological systems, the balance between prooxidants and antioxidants is crucial to cellular homeostasis. Since several studies have indicated the development of oxidative stress following liver toxicity [20,21], we examined the serum TAS level, which represent the major antioxidant systems of the body following INH/RFP administration. As observed in the present study, INH and RFP caused the impairment of liver antioxidant status, as evidenced by the significant decrease in TAS value. As is well known, cells have evolved an array of well- coordinated defense mechanisms comprising antioxidant molecules such as GSH, and antioxidant enzymes, all of which act synergistically to detoxify the oxidative injury by means of scavenging oxygen free radicals and represent the TAS status. Although INH and RFP are potentially hepatotoxic drugs, the precise molecular mechanisms behind their adverse effects are not fully understood. INH is metabolized to acetylisoniazid via hepatic N-acetyltransferase-2 [22]. In turn, acetylisoniazid is hydrolyzed to acetylhydrazine, which is oxidized by cytochrome P450 to form some hepatotoxic intermediates [23]. Moreover, several studies have proven that RFP increases INH toxicity, most probably by increasing the formation of its toxic metabolite hydrazine [24,25]. A logical approach to prevent INH/RFP-induced hepatic injury could be the potentiation of the antioxidant defense machinery of the host to guard the liver against these offending drugs. In the present study, Quercetin attenuates INH/RFP-induced oxidative stress, revealed as an increase in serum TAS value, as well as that of NAC-treated rats. Quercetin acts by scavenging the reactive metabolites and hydroxyl radicals and preventing their interactions with hepatic macromolecules [26]. In Quercetin-treated rats, the serum levels of liver enzymes markers were decreased significantly when compared with INH/RFP only treated group (group II) reverting back to normal levels. These results may support the hepatoprotective effect of Quercetin drug-induced toxicity [27]. In support of our present findings, several studies proved the protective effect of Quercetin on diabetes mellitus and improving insulin resistance $[28,29]$. The hepatoprotective effect of Quercetin on liver injury is well evident, which significantly inhibits the elevation of these enzymes levels in rats with ethanol-induced hepatotoxicity treated with quercetin, by keeping the structural integrity of the liver [30]. To our knowledge, this is the first study in which the protective effects of Quercetin against INH/RFP-induced hepatotoxicity through improving the antioxidant status and ameliorating inflammatory reactions have been reported. Such protection was detected both by diagnostic indicators of liver damage (AST and ALT levels) and by histopathological analyses. The oxidative injury that was induced by INH and RFP was substantiated by histopathological findings. The protection by Quercetin in this study was reflected by the absence of histological lesions. The present study further supported oxidative stress as the mechanism for INH/RFP-induced hepatotoxicity. The study presents a novel approach to prevent INH/RFP-induced liver injury by the coadministration of Quercetin. In conclusion, the hepatotoxicity due to long-term use of the anti-tubercular drugs, INH and RFP, can be effectively ameliorated by adjuvant use of Quercetin.

\section{Acknowledgement}

The authors thank the University of Sulaimani for supporting the project.

\section{References}

[1] Klaassen CD; (Ed.); Casarett and Doull's toxicology; the basic science of poisons;7th Edition;Mc-Graw Hill, New York; 2008; p. 557-576. 
[2] Nanashima K, Mawatari T, Tahara N, Higuchi N, Nakaura A, et al. Genetic variants in antioxidant pathway: risk factors for hepatotoxicity in tuberculosis patients. Tuberculosis, 92: 253-259. 2012.

[3] Santhosh S, Sini TK, Anandan R, Mathee PT. Hepatoprotective activity of chitosan against isoniazid and rifampicin-induced toxicity in experimental rats. Eur. J. Pharmacol., 572:69-73. 2007.

[4] Flanagan RJ, Meredith TJ. Use of N-acetylcysteine in clinical toxicology.Am. J. Med., 91(Suppl. C): 131S-139S. 1001.

[5] Brack C, Bechter-Thuring E, Labuhn M. N-acetylcysteine slows down aging and increase the life span of Drosophila melanogaster. Cellular Mol. Life Sci., 53(11-12): 960-966. 1997.

[6] Harwood M, Danielewska-Nikiel B, Borzelleca JF, et al. A critical review of the data related to the safety of quercetin and lack of evidence of in vivo toxicity, including lack of genotoxic/carcinogenic properties. Food Chem. Toxicol., 45: 2179-2205. 2007.

[7] Powers SK, DeRuisseau KC, Quindry J, Hamilton KL.Dietary antioxidants and exercise.J. Sports Sci., 22: 81-94. 2004.

[8] Reid MB. Free radicals and muscle fatigue: of ROS, canaries, and the IOC. Free Radic. Biol. Med., 44: 169-179. 2008.

[9] Jaeschke H. Preservation injury: mechanisms, prevention and consequences.J.Hepatol., 25:774-780. 1996.

[10] Georgieva N, Gadjeva V, Tolekova A. New isonicotinylhydrazones with SSA protect against oxidative-hepatic injury of isoniazid. TJS., 2: 37-43. 2004.

[11] Arsad SS, EsaNM, Hamzah H. Histopathologic changes in liver and kidney tissues from male SpragueDawley rats treated with Rhaphidophoradecursiva (Roxb.) schott extract. J CytolHistol., 4: 1-6. 2014.

[12] Robin MA, Le Roy M, Descatoire V, Pessayre D. Plasma membrane cytochromes-P450 as neoantigens and autoimmune targets in drug-induced hepatitis. J.Hepatol., 26(Suppl. 1):23-30. 1997.

[13] Yun CH, Okerholm RA, Guengerich FP. Oxidation of the antihistaminic drug terfenadine in human liver microsomes: role of cytochrome P-450 3A(4) in N-dealkylation and Chydroxylation. Drug Metab.Dispos., 21: 403-409. 1993.

[14] Trauner M, Meier PJ, Boyer JL. Molecular pathogenesis of cholestasis.N. Engl. J. Med., 339: 1217-1227. 1998.

[15] Reed JC. Apoptosis-regulating proteins as targets for drug discovery.Trends Mol. Med., 7: 314-319. 2001.

[16] Pessayre D, Berson A, Fromenty B, Mansouri A. Mitochondria in steatohepatitis. Semin. Liver Dis., 21: 57-69. 2001.

[17] Saad EI, El-Gowilly SM, Sherhaa MO, Bistawroos AE. Role of oxidative stress and nitric oxide in the protective effects of $\alpha$ lipoic acid and aminoguanidine against isoniazid-rifampicin- induced hepatotoxicity in rats.Food Chem. Toxicol., 48: 18691875. 2010.

[18] Chen $\mathrm{X}, \mathrm{Xu} \mathrm{J}$, Zhang $\mathrm{C}$, Yu T, Wang $\mathrm{H}$, et al. The protective effects of ursodeoxycholic acid on isoniazid plus rifampicin induced liver injury in mice. Eur. J. Pharmacol., 659: 53-60. 2011.

[19] Metushi IG, Nakagawa T, Uetrecht J. Direct oxidation and covalent binding of isoniazid to rodent liver and human hepatic microsomes: humans are more like mice than rats. Chem. Res. Toxicol., 25: 2567-2576. 2012.

[20] Yamamoto T, Kikkawa R, Yamada H, Horii I. Identification of oxidative stress-related proteins for predictive screening of hepatotoxicity using a proteomic approach. J.Toxicol. Sci., 30(3): 213-227. 2005.

[21] Jaeschke H, Ho YS, Fisher MA, Lawson JA, Farhood A Glutathione peroxidase-deficient mice are more susceptible to neutrophil-mediated hepatic parenchymal cell injury during endotoxemia: importance of an intracellular oxidant stress. Hepatology, 29(2): 443-450. 1999.

[22] Leiro-Fernandez V, Valverde D, Vazquez-Gallardo R, BotanaRial M, Constenla L, et al. N-acetyltransferase-2 polymorphisms and risk of anti-tuberculosis drug-induced hepatotoxicity in Caucasians. Int. J.Tuberc. Lung Dis., 15:1403-1408. 2011.

[23] Tostmann A, Boeree MJ, Aarnoutse RE, de Lange WC, van der Ven AJ, et al. Antituberculosis drug-induced hepatotoxicity: concise up-to-date review. J. Gastroenterol.Hepatol., 23: 192-202. 2008.

[24] Zhang ZH, Tang JH, Zhan ZL, Zhang XL, Wu HH, et al. Cellular toxicity of isoniazid together with rifampicin and the metabolites of isoniazid on QSG-7701 hepatocytes. Asian Pac. J. Trop. Med., 5: 306-309. 2012.

[25] Yew WW, Leung CC. Anti-tuberculosis drugs and hepatotoxicity. Respirology, 11: 699-707. 2006.

[26] Yousef MI, Omar SA, EI-Guendi MI, Abdelmegid LA. Potential protective effects of quercetin and curcumin on paracetamolinduced histological changes, oxidative stress, impaired liver and kidney functions and hematotoxicity in rat.Food Chem. Toxicol., 48: 3246-3261. 2010.

[27] Tolman KG. The liver and lovastatin.Am. J.Cardiol., 89: 13741380. 2002.

[28] Rivera L, Moron R, Sanchez M, Zarzuelo A, Galisteo M. Quercetin ameliorates metabolic syndrome and improves the inflammatory status in obese zucker rats. Obesity, 16: 2081-2087. 2008.

[29] Kobori M, Masumoto S, Akimoto Y, Takahashi Y. Dietary quercetin alleviates diabetic symptoms and reduces streptozotocin induced disturbance of hepatic gene expression in mice. Mol. Nutr. Food Res., 53: 859-868. 2009.

[30] Chen X. Protective effects of quercetin on liver injury induced by ethanol. Pharmacogn. Mag., 6: 135-141. 2010. 